\title{
Indentation : fondements, développements théoriques et expérimentaux
}

Ce numéro thématique de Matériaux et Techniques est composé d'une sélection d'articles, pour l'essentiel issus et rédigés à partir de communications présentées lors de la conférence «Indentation 2012 » qui s'est déroulée à l'École Centrale de Lyon du 29 au 31 octobre 2012. Ce colloque a reçu le soutien scientifique et financier du Labex Manutech SISE, de l'Institut Carnot Ingénierie@Lyon, de l'École Centrale de Lyon et du Laboratoire de Tribologie et Dynamiques des Systèmes. Cette conférence fait suite aux manifestations suivantes :

- 2000 : Lille, Journée de l'ATTT « contribution de l'essai d'indentation à la caractérisation des matériaux métalliques $»$;

- 2003 : Angers, Séminaire « Nanoindentation »;

- 2004 : Nancy, JMC9 Minicolloque « Micro et nanoindentation »;

- 2005 : Angers, Journées thématiques de la SF2M «Surfaces et interfaces, indentation rayage et abrasion » (Surfaces et interfaces, indentation, rayage et abrasion, Eds. S. Benayoun and E. Le Bourhis, Matériaux et Techniques, Volume 93, No. 7-8, 2005);

- 2008 : Rennes, « Colloque National sur l'Indentation» (Indentation : analyses, développements et applications, Eds. O. Bartier and G. Mauvoisin, Matériaux et Techniques, Volume 96, No. Hors Série, 2008);

- 2010 : Nantes, symposium intitulé «Indentation à différentes échelles » lors de la conférence « Matériaux 2010 » (Indentation à différentes échelles, Eds. E. Le Bourhis and G. Mauvoisin, Matériaux et Techniques, Volume 99, No. 2, 2011).

Ces évènements ont permis de fédérer une communauté francophone dont les travaux de recherche portent sur l'indentation et qui s'est structurée depuis 2009 au sein du Groupe Indentation Multi-Echelle (GIME) de la SF2M, Société Française de Métallurgie et de Matériaux (http://sf2m.asso.fr/CommissionsThematiques/Indentation.htm).

Cette conférence, dont le comité d'organisation était composé de S. Bec, S. Benayoun, G. Kermouche, J.L. Loubet et S. Pavan, a réuni 68 participants. Quatre conférences invitées (M. El Mansori «L'usinage abrasif des surfaces fonctionnelles et structurées par indentation dynamique multi-échelle », J. Michler « Essai nanomécanique in-situ en température pour l'exploration des mécanismes de fissuration et de plasticité dans les semi-conducteurs et les métaux », T. Pardoen «On chip testing of thin films» et M. Vandamme « Caractérisation du fluage par nanoindentation et application aux matériaux cimentaires »), trente exposés et une vingtaine de posters ont été présentés.

La simplicité apparente du test d'indentation, plus particulièrement dans sa mise en œuvre, a conduit à une utilisation très large de cet essai mécanique, tant en milieu industriel qu'en laboratoire de recherche. D. Tabor dans son ouvrage «Hardness of Metals » en 1951, présente une synthèse de travaux qui posent les fondements de l'interprétation et de la modélisation des mécanismes de déformations générés par l'indenteur. Il propose aussi de relier les résultats des essais d'indentation avec ceux des essais de compression uniaxiale sur les métaux testés, en introduisant notamment la notion de déformation représentative, permettant ainsi de déterminer la loi de comportement du matériau par indentation. Toutefois, la complexité de ces mécanismes, la prise ne compte des différentes échelles d'indentation, l'hétérogénéité des matériaux et des régimes de sollicitation, etc. ont montré les limites de cette approche et rendent toujours d'actualité des travaux de recherche sur l'indentation.

Les différents numéros thématiques de Matériaux et Techniques (Vol. 93 No. 7-8, 2005, Vol. 96 HS, 2008 et Vol. 99 No. 2, 2011), témoignent de l'intérêt et de la vitalité de ce sujet de recherche pendant cette dernière décennie, autant que des nouvelles interrogations suscitées par les récents développements. 
Ce numéro thématique s'inscrit dans la continuité des numéros précédents et propose, sous la forme de synthèse bibliographique ou d'article spécifique, de développer des aspects fondamentaux de la modélisation de l'indentation, de prendre en compte les essais d'indentation dans toute leur complexité en intégrant notamment l'hétérogénéité des matériaux, de montrer des applications non conventionnelles de l'indentation ainsi que d'étendre les approches développées pour l'indentation à la compréhension d'autres mécanismes tels que le rayage et les processus abrasifs. Ainsi, ce numéro propose des articles qui traitent de la notion de déformation représentative, ou encore de l'effet de la rugosité et des contraintes résiduelles. Un article s'intéresse aux caractéristiques mécaniques de tribofilms déterminées par nanoindentation pour comprendre le comportement tribologique de couches minces nanocomposites. D'autres articles traitent des mécanismes de rayage en décrivant les effets des contraintes résiduelles et de la lubrification.

Pour conclure, nous souhaitons remercier tout particulièrement les membres du comité scientifique de cette conférence, ainsi que toutes les personnes qui ont participé au comité de relecture des articles publiés dans ce numéro.

Les éditeurs invités

Sandrine Bec et Stéphane Benayoun 